http://jmscr.igmpublication.org/home/

ISSN (e)-2347-176x ISSN (p) 2455-0450

crossref DOI: https://dx.doi.org/10.18535/jmscr/v8i9.10

\title{
Eaton Embolism - Coexistent Pneumonia and Pulmonary Embolism
}

\author{
Authors \\ Dr Sahana K ${ }^{1}$, Prof Dr Meenakshi.N², Prof Dr Aruna Shanmuganathan ${ }^{3}$, \\ Dr Nisha Ganga ${ }^{4}$ \\ ${ }^{1}$ Postgraduate, ${ }^{2}$ Professor \& HOD, ${ }^{3}$ Professor, ${ }^{4}$ Senior Resident \\ Department of Respiratory Medicine, Chettinad Hospital and Research Institute, Kelambakkam, \\ Chennai 603103, India
}

\begin{abstract}
The coexistence of pulmonary embolism (PE) and pneumonia is known as infarction pneumonia. This condition is relatively rare in adults in comparison to children. The diagnosis of coexistent pneumonia with embolism is challenging as clinical features of fever, pleuritic chest pain and breathlessness maybe common in both conditions. Hence, the presence of coexisting embolism in pneumonia needs a high degree of clinical suspicion. We hereby report a case of a young woman who presented with Mycoplasma pneumonia and ARDS with early diagnosis of concommittant pulmonary embolism. Early recognition and management of both the conditions resulted in a favourable outcome.
\end{abstract}

Keywords: Pulmonary embolism, Mycoplasma, Pneumonia, Infarction.

\section{Introduction}

Mycoplasma pneumoniae infections are one of the common etiologies of community-acquired pneumonia (CAP). The Manifestations and clinical features vary widely and systemic involvement is common. Diagnosis is very challenging because the clinico radiological findings of the classical pneumonia is lacking. Moreover, Mycoplasma pneumonia is not routinely tested in the clinical setup. Extrapulmonary manifestations like Arthralgia, pancreatitis, transverse myelitis and severe pulmonary manifestations can lead to long-term sequelae. The treatment becomes more complicated with the increasing emergence of Mycoplasma resistance. The current case highlights coexistence of mycoplasma pneumonia and pulmonary embolism and need for early diagnosis and management and can be fatal if not diagnosed and treated early.

\section{History}

A 27 year old female, IT professional by occupation came with complaints of high grade fever, cough with mucopurulent expectoration and progressively worsening breathlessness for 1 week. She denied h/o Chest pain /hemoptysis/ wheezing .There was no prior history of any respiratory illness.. No comorbid illnesses. No significant Family history.

Clinical Examination revealed the patient was conscious, oriented, febrile, dyspnoeic and Tachypneic at rest. Patient was not anemic, non icteric, no Clubbing/ cyanosis/ pedal edema. JVP was not elevated. 
Vitals showed Spo2-93\% in room air, PR-145 beats/min, RR-22 cycles/minute, BP- 110/70 $\mathrm{mmHg}$, BMI- $22 \mathrm{~kg} / \mathrm{m}$

RS-Normal vesicular breath sounds with reduced intensity in right and left infraaxillary and infrascapular areas with bilateral fine basal crepitations
CVS- S1 S2 heard, no added sounds. Other systems normal.

Course in the Hospital: Patient came with above said complaints, in view of desaturation, patient was shifted to ICU and following investigations were done.

\section{Investigations}

\begin{tabular}{l}
\hline Routine Blood tests-:Hb - 8.1 gm\% \\
TC, DC, RBS, LFT, RFT, HIV - Normal \\
Sputum for AFB - Negative \\
Mycoplasma IgM Antibody -positive \\
ANA-positive, Coomb's test- positive \\
$\mathrm{ABG}-\mathrm{PaO}_{2}-78 \mathrm{mmHg}, \mathrm{PaCO}_{2}-43 \mathrm{mmHg}, \mathrm{Ph}-7.38$ \\
CXR PA(pre- treatment) -Bilateral non \\
homogenous opacity in the right and left lower zones \\
with obliteration of both costophrenic angles and \\
cardiophrenic angles. \\
CECT Chest- Consolidation of bilateral lower lobes \\
with atelectasis and bilateral pleural effusion \\
CTPA- Thrombus involving segmental and \\
subsegmental branches of posterobasal segment of \\
Right pulmonaryartery. Partial thrombus involving \\
lateral basal segmental branches of Left \\
pulmonary artery \\
F.O.B -Complete narrowing of left upper lobe \\
bronchus with inflammed mucosa \& mucoid \\
secretions \\
(BAL for AFB \& gene expert - negative) biopsy \\
shows no granuloma and malignancy \\
PFT - Small airway obstruction.
\end{tabular}

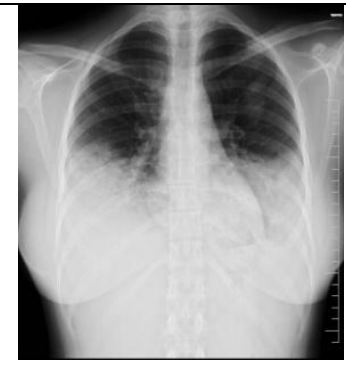

PRE- Treatment xray POST-Treatment xray

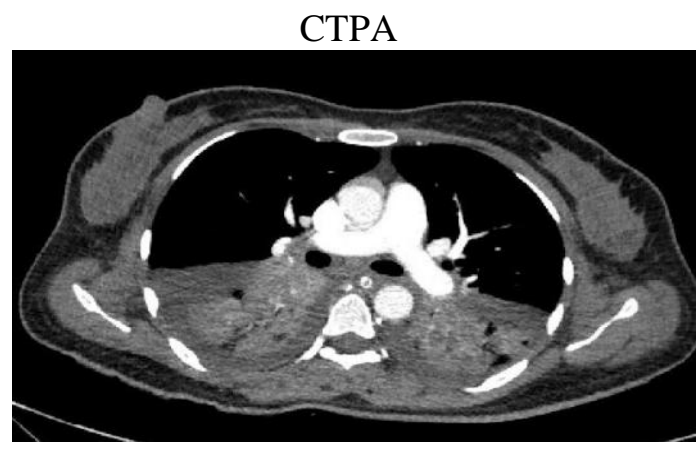

\section{Results \& Treatment}

\section{CECT Chest}
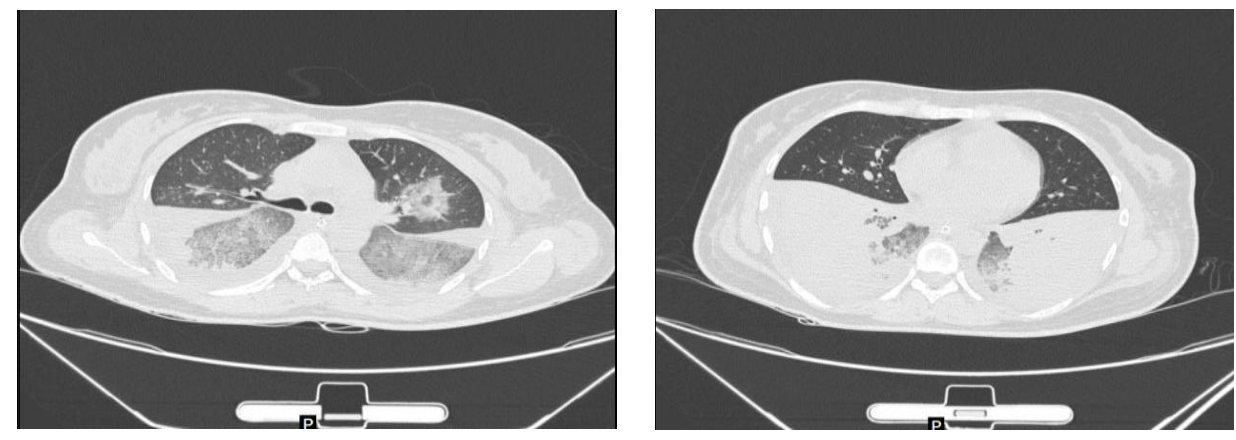
CTPA
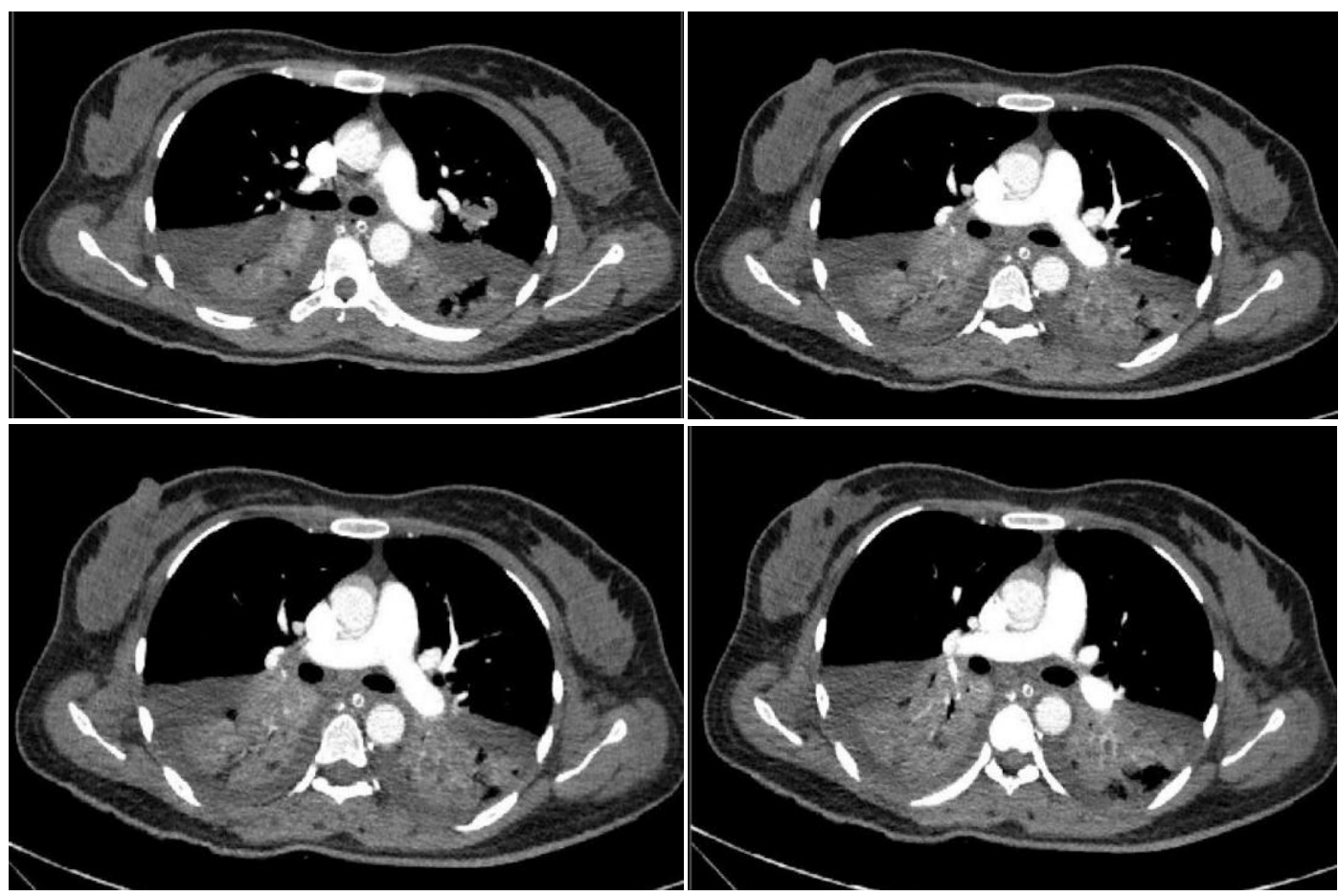

\section{Treatment}

Patient clinically presented with signs and symptoms of pneumonia and ARDS and was initially started on broad spectrum antibiotic. In view of worsening hypoxemia, patient intubated following a trial with NIV. Since patient had persistent tachycardia and pleural based lesion, pulmonary embolism was suspected and CTPA was done and revealed thrombus involving segmental and subsegmental branches of posterobasal segment of Right pulmonaryartery. Partial thrombus involving lateral basal segmental branches of Left pulmonary artery. All cultures were negative but Mycoplasma IgM antibody was positive, Patient was started on anticoagulants and Clarithromycin. Patient significantly improved with treatment and discharged with oral anticoagulants and Antibiotics.

\section{Discussion}

Mycoplasma pneumonia accounts for $7-20 \%$ of community acquired pneumonia. Community acquired pneumonia causing pulmonary embolism is known as infarction pneumonia which is the most important cause for mortality. It can cause hemophagocytic syndrome which leads to disruption of vessel wall integrity resulting in pulmonary embolism and ARDS. The probable pathophysiology postulated in our case is Mycoplasma vasculitis leading to pulmonary embolism.

Pathogenesis can be either direct or indirect type. The mechanism of direct pathogenesis is that mycoplasma pneumoniae blood-borne metastasis may induce cytokines such as tumor necrosis factor- $\alpha$, chemotactic factor and interleukin- 8 which affect vessel wall and lead to vascular occlusion or local angiitis. The indirect pathogenesis is that mycoplasma pneumoniae causes immunologic derangement which produces phospholipids and $\operatorname{IgM}$ anticardiolipin antibodies to form a temporary hypercoagulable state, leading to deep vein thrombosis. These findings reflect a systemic infection-related prothrombotic states. The clinical features for infection is chills, purulent sputum, or bacteremia, whereas pulmonary embolism is confirmed by CTPA. Therefore, the possibility of pulmonary infarction should be considered when there is continued clinical and radiological worsening in case of pneumonia despite treatment with antibiotics and optimal management. 


\section{Conclusion}

This case highlights the importance of suspecting pulmonary embolism in pneumonias with atypical presentation not responding to standard care of management. A high clinical suspicion and early diagnosis of coexisting pulmonary embolism prevents complications and mortality.

\section{References}

1. Graw-Panzer KD, Verma S, Rao S, Miller ST, Lee H. Venous thrombosis and pulmonary embolism in a child with pneumonia due to Mycoplasma pneumoniae. Journal of the National Medical Association. 2009 Sep 1;101(9):956.

2. Chan ED, Welsh $\mathrm{CH}$. Fulminant Mycoplasma pneumoniae pneumonia. Western journal of medicine. 1995 Feb;162(2):133.

3. Venkatesan P, Gladman J, Macfarlane JT, Barer D, Berman P, Kinnear W, Finch RG. A hospital study of community acquired pneumonia in the elderly. Thorax. 1990 Apr 1;45(4):254-8.

4. Narita M. Pathogenesis of extrapulmonary manifestations of Mycoplasma pneumoniae infection with special reference to pneumonia. Journal of infection and chemotherapy. 2010 Jun 1;16(3):162-9.

5. Morichika K, Nakachi S, Tomoyose T, Masuzaki H. Thromboembolism supervened on Eosinophilia induced by Mycoplasma Pneumonia. Internal Medicine. 2016 Sep 15;55(18):2741-2.

6. Su HY, Jin WJ, Zhang HL, Li CC. Clinical analysis of pulmonary embolism in a child with Mycoplasma pneumoniae pneumonia. Chinese journal of pediatrics. 2012 Feb;50(2):151-4.

7. Flateau C, Asfalou I, Deman AL, Ficko C, Andriamanantena D, Fontan E, Viant E,
Bonnevie L, Rapp C. Aortic thrombus and multiple embolisms during a Mycoplasma pneumoniae infection. Infection. 2013 Aug 1;41(4):867-73.

8. Paparoupa M, Spineli L, Framke T, Ho H, Schuppert F, Gillissen A. Pulmonary embolism in pneumonia: still a diagnostic challenge? Results of a case-control study in 100 patients. Disease markers. 2016 Jan 1;2016. 